\title{
Using Digital Coaching to Promote Physical Activity to University Students with Low Levels of Physical Activity: A Qualitative Intervention Study
}

\author{
Eeva Kettunen \\ University of Jyväskylä \\ eeva.k.kettunen@jyu.fi
}

\begin{abstract}
Physical activity levels of university aged people have been declining in recent years. This age group is experienced in incorporating technology into their lives. Therefore, it is worthwhile to study the role sport and wellness technology could play in health promotion to this group. This exercise psychology focused intervention study explored the effects, needs and wants related to using a digital sport and wellness technology digital coach in the pursue of a more physically active lifestyle. The target group of this study was university students with low levels of physical activity. The results suggest digital coaching can motivate people with low levels of physical activity by giving them better selfawareness related to exercising. The individualized training program and feedback was perceived as positive, and digital coaching could be more effective with development towards digital coaching having more 'human' elements. These results provide information for companies and professionals working in sport and health promotion.
\end{abstract}

\section{Introduction}

University age students in general have been found to be at a high risk of disengagement from physical activity [1]. Insufficient physical activity is a serious health concern among this population [2]. Furthermore, according to World Health Organization (WHO) physical inactivity is a global problem having detrimental effects on health [3]. Therefore, it is important to explore new ways to help people become more physically active.

University students can be considered the "net generation" - people born after 1977 [4] - who have used different kinds of technology throughout their entire lives. With their familiarity with technology and integration of it into different parts of their lives, it is worthwhile studying whether using technology for exercise promotion purposes has potential to motivate university students toward a more physical active and healthy lifestyle.

Sport and wellness technology devices and applications have recently become more popular. Previously, these devices were prohibitively expensive, used only by the most dedicated exercisers. In the past 10-15 years the devices have become significantly less expensive and are more widely used by regular exercisers. According to previous research, receiving feedback on one's performance is considered more relevant and effective during the beginning of skill acquisition [5]. Considering this, sport and wellness technology devices and applications are also suitable for recreational exercisers with a low or intermediate knowledge level and are in the initial level of skill acquisition [6].

Even though sport and wellness technology has potential to increase the quality of physical activity and training via personalized feedback, using technology may also lead to inappropriate adjustments to training, particularly as a result of misinterpretation by the user [7]. Therefore, the information should be easy to understand and implement and importantly, matches the user's level of understanding. Providing too much information, unnecessary information, or inadequate information can create stress and anxiety for the user [8].

According to previous research, receiving feedback on how to maintain or enhance the level of physical activity and overall wellness can make users more goal oriented [e.g., 9, 10], which can lead to an increase in motivation $[11,12]$. When looking at many current sport and wellness technology applications and devices, they focus only on giving feedback data from past performances instead of focusing on giving instructions and personalized suggestions on what to do next in order to get closer to personal goals.

A solution for this problem is digital coaching, a "service on a technological device that not only gives feedback but also offers advice, suggestions and future steps for a user to follow in the pursuit of their wellness 
and fitness goals" [13 pp.3]. While typical sport and wellness technology devices and applications only give feedback on performance data, a digital coach creates a personalized training plan. A digital coach can potentially identify the weaknesses and strengths of a user, and based on the collected data, create a personalized training plan and update it based on the user's actions [14]. It is important to acknowledge that digital coaching does not refer to the use of digital tools by human coaches, rather, it refers to a device or solution which in itself is the coach and functions without a human coach. The potential a digital coach has in exercise and physical activity settings has been recognized in previous IS research $[15,13,16]$.

Interest in developing sport and wellness technology towards being more personalized and instructional is becoming more popular. However, digital coaching commercial solutions and devices are still relatively new in sport and exercise settings. Few studies have focused on usage experiences of digital coaching devices and applications. This study continues studying the effects of digital coaching by focusing on university students with low levels of physical activity. The study looked at the topic mostly from an exercise psychology perspective focusing on motivation and self-efficacy. Observing how using a digital coach can affect the participants' understanding and opinion about physical activity can help to understand how to create a basis for long lasting exercise motivation. The study also highlights some issues related to user experiences to understand how to further develop digital coaching.

The research questions for this qualitative study are the following: 1. How can sport and wellness technology digital coaching influence university students with low physical activity when trying to become more physically active? and 2 . How would they describe their experience of using a digital coach and what are the perceptions, suggestions and wishes regarding digital coaching solutions in general? University students with low levels of physical activity were selected as a target group since they represent a demographic that are potential customers of digital coaching devices and solutions. This study contributes by increasing the theoretical and practical understanding on the usage experiences of digital coaching type of sport and wellness technology devices and applications. The study also brings insight for the developers of these types of technologies.

\section{Theoretical background}

The theoretical framework used in this study is the social cognitive theory by Bandura [17]. This theory is frequently used as a framework when examining motivation and physical activity. As part of social cognitive theory, Bandura introduced the concept of "self-efficacy", referring to how a person perceives their own capabilities in performing a task. People with high self-efficacy may be more likely to see a difficult task as a challenge, while those with low self-efficacy may see challenging tasks as too difficult and avoid them entirely [17]. A person's self-efficacy can be affected by a variety of information provided to them, those sources being generally categorized into four groups: previous performance accomplishments, vicarious experiences, verbal persuasion, and physiological states [17]. A person's self-efficacy is also linked to their motivation, since tasks that are considered too difficult or too easy when compared to their own perceived skill level may decrease motivation, and those which are moderately challenging yet achievable may increase motivation [18]. People with high self-efficacy may also put in more effort, be more persistent, and participate more often in physical activity [17]. Thus, self-efficacy has an important role in exercise.

Referring again to motivation, another key theory is the theory of self-determination (SDT) [19]. This theory highlights three "needs" that can affect motivation: the need for autonomy, for competence, for relatedness. Briefly, the need for autonomy relates to being able to self-regulate their behavior. The need for competence refers to being able to complete a task by effectively interacting with the surrounding environment. The need for relatedness refers to connecting with others. Each of these "needs" can, either individually or together, facilitate intrinsic motivation. Intrinsic motivation generally refers to receiving motivation and satisfaction from performing the activity itself, rather than requiring additional external stimuli or rewards. In the context of physical activity and sports, having high intrinsic motivation has been connected with forming positive exercise habits and a physically active lifestyle.

Recent research aimed to create a model for designing human-computer interaction solutions that support sustained engagement, behavior change, and wellbeing of users. Peters et al., [37] introduced the "Motivation, Engagement and Thriving in User Experience" (METUX) model, building on the selfdetermination theory to consider user outcomes of engagement, motivation, and thriving. The outcomes are mediated by the three factors of self-determination, which are impacted by the design of the product. The creation of a model like this shows the growing importance of the design of technological products in fostering engagement, behavior change, and wellbeing.

Another concept derived from Bandura's social cognitive theory is that of "proxy agency" [20]. Proxy agency suggests that people use "agencies" to assist in their self-development, self-renewal, and adaptation to life's demands. An "agency" refers to acts done with a 
specific purpose, and Bandura defines three forms: personal, proxy, and collective. In personal agency, the person themselves acts upon the things they can personally control. Proxy agency refers to situations where a third party (an agent) will act upon the person's behalf. In collective agency a group of people pool their resources together $[20,21]$, as would be the case in a group project or team of athletes or exercisers.

According to Bandura [20] there are three reasons why a person would utilize proxy agency. First, a person may feel as though they do not have requisite skills or knowledge to reach their goal. Second, they may perceive that someone else is more capable of bringing them closer to their desired goal. Third, although a person may have the needed skills and knowledge, they may wish to transfer control (and responsibility) to someone else [22]. In physical activity settings, using a proxy agent, like a personal trainer, can help a person manage their task demands and assist in the regulation and control of the desired exercise behavior, and may provide instruction on learning new skills and social support. These may increase the likelihood of the person being more involved, focused, and having increased enjoyment. Proxy agency may influence an athlete's self-efficacy by providing vicarious experiences through observation,by using verbal persuasion, evaluation and feedback, setting expectations, or the use of imagery, all of which are widely used by trainers and coaches [23].

In this study, the proxy agency theory is examined from the point of view of a digital coach, where the concept of a proxy agent refers to some sort of sport and wellness technology that has a digital coaching feature. A digital coach attempts to mirror the behavior of a human proxy agent, by influencing feelings of competence by providing feedback and instructions, using a variety of persuasion techniques, in addition to other typical sports watch features.

Some research has suggested that long-term use of a proxy agent can lower the self-regulatory skills of a user, which are an important aspect in the independent management of sport and physical activity behavior [25]. However, there is limited research studying the effects of using non-human physical activity proxy agents. A digital coach, when used as a proxy agent may be a better choice than a human proxy agent in this sense, however, as a digital coach still requires a certain amount of independence and regulatory skills, while still providing some proxy agency. This may also positively affect feelings of autonomy. However, because the use of a digital coach also turns over more responsibility to a user, concerns related to the appropriate delivery and interpretation of the information become more present.

\section{Methodology}

\subsection{The digital coach used in this study}

The device used in this study was the Suunto 3 Fitness sports watch, created by Suunto OY based in Finland [26]. The device is marketed as a training watch for exercisers, and is particularly for running/walking and cycling. The Suunto 3 Fitness features "adaptive training guidance", making the watch serve as a digital coach. The device creates a 7-day training plan based on the user's fitness level and goals.

The user may also select a fitness goal, which affects the amount of recommended exercise. As a user's fitness level changes, the recommendations also change to be both challenging and achievable. The specific workout for each day is based on the user's recent training history and the remaining training load goal for the rest of the week. Background rules on the watch ensure appropriate training recommendations. For example, a hard workout is never recommended on two consecutive days.

The plan is presented on the screen, and a user can view each workout individually. On the day a user plans to exercise, the watch provides the workout goals, typically as a time measure, and an intensity description, such as "easy", "moderate", or "hard". During the workout, real-time guidance is given to ensure proper exercise intensity is achieved, using watch alarms instructing the user to speed up or slow down.

After the workout, the device provides text and numeric feedback, such as distance travelled, time spent in different heart rate zones, and estimated time needed for full recovery. Subsequent workouts may be changed based on the outcome of this workout, to avoid challenging the user too much. Alternatively, the training guidance will also adapt if a person misses a workout or decides to not do the recommended workout.

\subsection{Data collection and analysis}

The study followed a qualitative approach. The study included 30 participants who were given a sport and wellness technology digital coach for a 10-12 weeks period. The study was conducted in Finland during autumn and winter 2018-2019. The target group of the study was university students with low levels of physical activity but who expressed their want to increase their physical activity level. The participant recruitment was done via the student online magazine of the author's university inviting all students to take part in the study who categorized themselves being passive or currently non-regular active exercisers but who wanted to increase the level of physical activity. 
Participation was on a voluntary basis as it was desired that all participants had at least an initial interest towards sport and wellness technology digital coaching. Initial interest was considered important since, like in real life, sport and wellness technology digital coaches will be bought and used only by people who are interested in the devices and solutions.

In total, 49 students expressed interest in taking part in the study. The students who, based on their personal descriptions about their current exercise habits, met the criteria of low or sedentary physical activity level were taken into the study until 30 participants were recruited. All selected participants had very low levels of current physical activity, but they were interested in finding a way to get them more motivated. The criteria for low levels of physical activity were based on the UKK Institute which is a center for health promotion research in Finland. The weekly recommendations of the UKK Institute are minimum $2 \mathrm{~h} 30$ min moderate exercise or $1 \mathrm{~h} 15 \mathrm{~min}$ vigorous exercise per week [27]. Participants did not meet these requirements. Participation was limited to 30 due to the number of available digital coach devices. All participants were provided the digital coach device by the author for the use period of the study - 10-12 weeks. In the beginning of the study the participants were given an introduction to the main functions of the digital coach and the watch itself. The users were asked to include the digital coach in their exercising and daily life in a manner that best suited them. By not giving any strict guidelines for usage the aim was to see how the participants naturally integrated a digital coach into their daily life. More detailed information about the participants can be seen in the table 1 below. The physical activity categories shown in the table derived from the Finnish National Sport survey [28] and include, ordered from the highest physical activity level to the lowest physical activity level, the following: competitive athlete, recreational sportsman, physically active for fitness, physically active for health, active in commuting and non-exercise, occasionally active, and sedentary.

Table 1. Participant information

\begin{tabular}{|l|c|c|c|}
\hline & Male & Female & Total \\
\hline $\mathbf{N}$ & 10 & 20 & 30 \\
\hline Age & & & \\
\hline$<25$ years & 1 & 7 & 8 \\
\hline $25-30$ years & 1 & 7 & 8 \\
\hline $31-35$ years & 4 & 2 & 6 \\
\hline $36-40$ years & 3 & 2 & 5 \\
\hline$>40$ years & 1 & 2 & 3 \\
\hline
\end{tabular}

\begin{tabular}{|l|c|c|c|}
\hline Physical activity level & & & \\
\hline $\begin{array}{l}\text { Physically active for } \\
\text { health }\end{array}$ & 1 & 3 & 4 \\
\hline $\begin{array}{l}\text { Active in commuting } \\
\text { and non-exercise }\end{array}$ & 6 & 10 & 16 \\
\hline Occasionally active & 3 & 6 & 9 \\
\hline Sedentary & 0 & 1 & 1 \\
\hline Degree under study & & & \\
\hline Bachelor's degree & 3 & 5 & 8 \\
\hline Master's degree & 6 & 14 & 20 \\
\hline Doctoral degree & 1 & 1 & 2 \\
\hline Study mode & & & \\
\hline Full-time student & 6 & 15 & 21 \\
\hline Part-time student & 3 & 4 & 7 \\
\hline Other & 1 & 1 & 2 \\
\hline
\end{tabular}

The data collection was done via semi-structured interviews. A semi-structured interview does not include a complete script, but instead a pre-formed structure that the interviewer follows [29], which was created for this study. The interview script comprised of the following sections: 1. Background information, 2. Expectations for digital coaching, 3. User experiences, 4. Adaptive training guidance and real-time feedback, 5 . Effects of the digital coach on exercising and 6. Ideal digital coach. For the sections 2-6 some of the questions were designed in a way to focus on the motivation and self-efficacy related issues of the topic.

All 30 participants were interviewed separately after 10-12 weeks of usage experience. In total, the average interview length was 48 minutes and varied from 36 to 69 minutes. The data analysis started by getting familiar with the data and transcribing the relevant parts of the interviews under each interview sections. The thematic analysis method was chosen for this study. This method is used for "analyzing, identifying and reporting patterns within data" [30, pp. 79] and is the most widely used analysis method in qualitative research [31]. In this research, thematic analysis enabled identifying, studying and comparing the occurring themes of the data set. Thematic analysis organizes and describes the data in rich detail interpreting various aspects and expectations related to the topic. The guidelines of Braun and Clarke [30] were followed during the analysis phase. These guidelines were applied in a flexible manner to fit the research questions and data. In the analysis process, a recursive process, rather than a linear phase-to-phase process was used, allowing moving back and forth between the analysis phases and reaching a deeper understanding 
and more detailed analysis of the entire research data. In the analysis the focus was on one interview section at a time. Each person's answers were written in an Excel file which enabled searching for reoccurring themes, similarities and differences between participants related to the same topic. The most highlighted themes of each section are reported in this paper.

\section{Results and findings}

\subsection{User experiences}

In general, the participants felt the digital coach was easy to learn to use. The only problem some users faced in the beginning of the test period was related to synchronizing their phone with the application which then would provide them with their baseline fitness level used in creation of the personalized training program. 23 of the 30 participants reported using the digital coach for the entire study period and the remaining 7 participants started using the device but then quit using it entirely after 1-2 months. The reasons for stopping was usually due to wearing the watch did not feel comfortable or the participants no longer found the device useful for them. However, some participants said they stopped using the device because they had already learned a lot and felt they did not need it anymore. Half of the participants reported having worn the digital coach also during night time, which provided them information also about their sleep. The other half used it only during the daytime.

The digital coach had several different features described before. The most preferred and utilized features of the digital coach were the adaptive training guidance, training diary and step counter. Almost as popular features were sleep measurement, stress and recovery measurement as well as heart rate measurement. Participants appreciated the digital coach having a wide variety of sports where to choose from and this inspired couple of participants to even try new types of exercising. The digital coach also kept participants up to date on their current stress and energy levels. Most of the participants paid attention to their stress level score but only a few participants ever modified their daily life based on their stress score. Nevertheless, paying attention to their stress score made some participants think about the reasons behind the score and what might cause them stress: "I learned to recognize what kind of situations can lower my stress level and how those situations change my feeling at the time". Many participants reported that they did not necessarily believe that they were stressed even though the digital coach said so. One of the reasons for this disbelief was the lack of understanding on what the stress score is based on. Most of the participants also appreciated the fact that the digital coach could also been used as a basic watch that not only shows time and date but can also be synchronized with messages and emails if wanted. However, none of the participants wanted to share their training results on social media even though the application had easy options for doing so. The reason for not sharing was usually that participants felt exercising and exercise data is person and they did not want to be compared to other people.

Participants were also asked about what they felt the positive and negative issues were related to the digital coach. Participants felt that the digital coach was easy to use and offered a large amount of data which helped participants understand more about their own exercising. Having visible data made them more aware about their exercising and encouraged them to be more physically active. As one participant said: "It is a convenient way to follow your own progress and see that little effort actually does already makes a difference when it comes to improving my fitness". Participants also liked the notifications received, such as when they achieved a daily step goal. In general, a positive thing was that the digital coach was able to increase the knowledge and understanding of the participants related to training and recovery.

Besides the positive experiences, participants also had negative experiences and suggestions on improving the digital coach. Some participants felt the digital coach should give more notifications when the user had missed a workout or had an otherwise less physically active period. Some participants felt that the device gave too much information, causing them more stress. Still, most participants would have liked to have more explanatory information supporting the result and suggestions the digital coach offered them. In other words, they felt the current level of communication was not always enough or sometimes not clear enough for the user to be able to learn from it. Another issue that was highlighted by many participants was the lack of trust in accuracy of the wrist heart rate measurement, which affected the usage experience of other features such as stress data, sleep data and adaptive training guidance.

\subsection{Adaptive training guidance and feedback}

In the beginning of the usage period the digital coach planned a personalized training plan for each participant. The plan was based on the personalized information received from the user as well as on their fitness test results. The first impressions about the program were fairly unanimous: the program seemed easy and not too demanding. However, based on the feelings associated with the first impression, the participants split into two. For the first half the easy start was seen as positive and encouraging. Since the trainings were relatively short in the beginning, for this 
group, it created a feeling that the goals are achievable. For half of the other participants, the easy start produced negative feelings. Having only a relatively short training session made these people doubt the effectiveness and usefulness of the training plan which lead to demotivation towards following the scheduled program. As one participant said: "I started laughing when I received instructions for trainings that lasted for 20min. When I used to actively train, my session lasted like 2 hours." The skepticism of the effectiveness of the program occurred especially with participants who had an exercise background despite being currently not being very physically active. However, some of these participants said that even though it does not feel natural for them, having a slow start would be smarter when starting to exercise after a break and therefore they understood the reasoning behind the program.

Participants were instructed to follow the adaptive training guidance the way that best suited them. All participants said they had become familiar with the training plan and tried it. However, 10 participants stopped using the training plan after the first try. 13 participants reported following the training plan but eight of them quit following it approximately after the half way of the study, whereas five participants reported using the adaptive training guidance throughout the entire study. The remaining participants used the training guidance occasionally. Most participants using the adaptive training guidance wished that they could have modified the training plan to better fit their everyday life. This modification usually meant switching training and rest days. One reason why some participants did not use the adaptive training guidance was that they wanted more personalization: "In order for me to be willing to give more control to the device in guiding my training, the digital coach would have needed to be even more personalized". However, some participants saw the adaptiveness of the program as a positive feature and were delighted that someone is planning their exercising for them.

A feature related to the adaptive training guidance was the real-time feedback. Eight participants reported not using the feature in their training sessions while the rest did. Reasons for not using the real-time feedback feature was usually mistrust of the heart rate measurement accuracy or a preference to follow one's own feeling rather than instructions related to pace and intensity. Most participants who included real-time feedback in their training session felt that the feature was helpful. The participants reported often changing their intensity based on the digital coach's instructions.

Overall, regardless of the level of usage, participants felt they gained something from using the adaptive training guidance as well as real time feedback. Participants felt the extra information they received not only helped them to train at the proper intensity level, but also that varying their intensity, such as through interval training, is also beneficial. Some participants said they learned to feel in their body what the right intensity level feels like: "I learned to recognize how I feel when my heart rate is at a particular level and because of this I can also train without sport technology in the future". 27 of 30 participants expressed at the end of the study their interest to use either adaptive training guidance, real-time feedback or both in the future.

\subsection{Effects on physical activity}

When participants were asked whether their physical activity had changed in some way during the test period, 22 participants reported having experienced a change. Most of them reported a slight increase in the daily activity due to the increase in every day active commuting. For some participants this meant switching driving or biking to walking whereas some participants increased the amount of walking in general. Some participants also reported being more consistent with their exercise and having had more variety on their physical activity. A few participants had started a new hobby as a result of being in the study. Four participants reported having better physical fitness after the study period whereas one participant said their physical condition had gone backwards during the study.

24 of the participants said using the digital coach had had a positive effect on their exercise motivation whereas only one person reported having negative effects. Seeing one's own exercise data and getting feedback on performance were the key issued that seemed to increase the level of motivation. Receiving personalized data and instructions seemed to make participants more aware of their own physical activity and health and the increased knowledge lead to positive motivation. As one participant said: "The digital coach helped me to channel my exercise motivation that I already had in me. Training became more consistent and since I started training at lower intensities my training motivation lasted longer."

However, in general participants highlighted that a digital coach cannot be the only source of exercise motivation. But, if a user has at least a small level of personal motivation, a digital coach can be a useful tool to help move exercising to the right direction. Participants could see digital coaching fitting many types of users from beginners to athletes. However, it would be most suitable for people who are interested in technology, receiving exercise data, and using that to reaching their physical activity goal. Most participants also recommended a digital coach for beginner exercisers who do not have information on how to start exercising and who do not have time, interest or courage 
to necessarily join group exercise or hire a personal trainer. Participants saw a digital coach having mostly positive effects on users but some highlighted that technology should not be the central focus while exercising so that it does not create stress.

Participants were also asked whether using the digital coach had any influence on their exercise selfefficacy. 9 participants reported having some positive effects on their exercise self-efficacy, often due to the performance data, increased knowledge and positive feedback. One participant felt an increase in selfefficacy because they realized they did not necessarily need any outside help but were capable of being in charge of their own exercising. None of the participants reported having any negative effects on their exercise self-efficacy. Some participants also reported that using digital coaching impacted their lives outside of exercising. They reported feeling more energetic during the day, which resulted from exercising more or going to bed earlier and gaining more sleep.

At the end of the study participants were asked whether they will change their physical activity habits in any way in the future due to the usage of the digital coach. 26 of the 30 participants said they have made changes to their exercising routines and will continue the new routine also in the future: "Since I noticed how bad shape I am in, I started making little changes towards a more active lifestyle, such as walking home from school." In most cases the reason for this change was the good feeling participants had received from exercising, suggesting the motivation had become more intrinsic. Some participants had bought their own sport and wellness technology device, some participants had booked a health check with their doctor and one participant had hired a personal trainer. Most participants said they will continue tracking their exercising also in the future in one way or another.

\subsection{The ideal digital coach}

At the end of the usage period the participants were asked to describe what their ideal digital coach would be like and how it would function. One third of the participants suggested their ideal digital coach would be a combination of a watch and mobile phone application. The next equally popular options were a watch and a mobile phone application as separate units. Some participants wanted their digital coach to be less visible such as a bracelet or a necklace. Some participants also would like to include a heart rate belt into their ideal digital coach to guarantee a more accurate heart rate measurement. The participants were also asked what type of information their ideal digital coach would provide them. The answers varied between participants but the most common features were related to overall training data and features like training intensity, training plan, or heart rate. Also, sleep and recovery related information was considered important as well as step and calorie counting. Food and nutrition related to training or weight loss was also considered essential. Other less common suggestions were that the ideal digital coach could have exercise route options, body temperature measurement, information on meditation and relaxation or psychological wellbeing, a weather forecast, drinking water consumption data. Some participants also wanted their ideal digital coach to include advice also on gym training and would be able to analyze whether the user can perform all the movements in a correct and safe way. The theme that was common is that the digital coaches should be able to adjust based on the users' personal needs and wants and that after purchasing a digital coach person should be able to modify the digital coach to include the essential features and if necessary, delete features they are not interested in. A digital coach should be easy to use and also have some human elements: "Manufacturers could make digital coaches more human like and less like a machine that encourages people to constantly complete tasks like a robot".

Participants reported that they preferably like to receive information from their ideal digital coach via text. Less common suggestions were voice, graphics, videos and numerical data. While training the ideal way of communicating with the digital coach would be sound and vibration since they were considered least disturbing. Some participants also suggested their ideal digital coach would have voice control, which would enable easy communication with the coach especially during the training. Participants also describe what kind of personality their ideal digital coach would have. Most participants wanted to have a positive and supportive and at the same time realistic digital coach who would remind them when they should exercise again. The feeling that the digital coach actually pays attention and is interested about the user was also highlighted.

Participants were also asked whether they would prefer, in the future, a digital coach or a personal human coach as their trainer. 22 participants chose a human coach whereas 7 participants chose digital coach. One participant would choose neither. The reasons for choosing a digital coach were the lower cost and the freedom to use the coach at their convenience. Another reason for choosing a digital coach was feeling less pressure as there was no other person involved to judge or make demands, making this option feel easier and less scary for some participants. One participant also highlighted that: "By having a digital coach you do work for yourself and not for someone else. And when you succeed you can be proud of yourself and take the full honor and responsibility". 
Reasons for selecting a personal human coach were related to the overall human interaction and the ability to communicate and be heard. A human coach could also see the person as a whole and make adjustments and corrections when needed. The participants appreciated the fact that they would be able to ask and get extra information from a human coach related to different topics and also talk about issues that do not necessarily relate to training. Participants described that the bond with a human coach compared to a digital coach would be tighter and they would be less likely to skip trainings as they feel they owe something to someone. However, most participants stated that if it were possible, they would prefer having both coaches, because the combination would support one another. The human coach could get information from the digital coach as well as help the user to use the digital coach more efficiently. On the other hand, during the times between meeting with a human coach, the digital coach would monitor and keep the user up to date on the progress.

\section{Discussion}

The purpose of this study was to explore the effects, needs and wants related to using a sport and wellness technology digital coach in the pursuit of a more physically active lifestyle. The target group was university students with low levels of physical activity.

The study examined the topic from an exercise psychology perspective focusing on motivation and self-efficacy but also focusing on issues related to user experiences. The research questions were: 1. How can sport and wellness technology digital coaching influence university students with low levels of physical activity when trying to become more physically active? and 2. How would they describe their experience of using a digital coach and what are the perceptions, suggestions and wishes regarding digital coaching solutions in general?

The findings indicate that university students with low levels of physical activity do find sport and wellness technology digital coaching interesting in general and feel a digital coach may help them become more physically active. Digital coaching can provide a large amount of personalized data, which might have otherwise been unavailable, and was found useful and motivating. This finding supports previous research findings [32, 33]. However, user's background knowledge is an important consideration. An expert exerciser may appreciate raw data, novice users need data that is easy to understand and act upon. Users also desire to increase their knowledge level of their own physical activity and health and would have liked to get even more information from the digital coach on topics they were interested in. With the specific device in this study, the topics participants followed the most were the adaptive training coach, training diary and step counter.

The real-time feedback from the device was perceived positively, helping users to find optimal exercise intensity and bring some variation into training. The adaptive training guidance feature that builds upon the real-time feedback was viewed as interesting, but participants tended to stop using the feature after some time. The adaptive training coach offered relatively easy recommendations at first, which has been shown by research to decrease barriers to starting and increases motivation. Conversely, some participants disliked how initially easy it was, which increased mistrust in the guidance and decreased motivation to use it.

In general, digital coaching was seen to have potential for people with low levels of physical activity and who are interested in technology and data. However, a digital coach by itself may not be able to motivate users to exercise.

From a theoretical perspective, the results show that digital coaching can affect users' exercise motivation by making training more goal oriented and also by increasing the overall knowledge with personalized data. Increased knowledge can increase self-efficacy. Having a relatively easy start in the personalized training plan can also increase self-efficacy by goals that seem achievable. These results support the results of a previous study [34] which highlighted that increased self-efficacy related to the users' confidence in their abilities to overcome obstacles related to exercising and their ability to train independently without support from friends, family or a trainer.

From the point of view of self-determination theory, the feeling of competence is the most important factor affecting the motivation of people with low levels of physical activity. The feeling of relatedness was also highlighted since participants liked communicating with the digital coach and appreciated that they were being provided support for their exercise, such as by having workouts planned for them. The importance of relatedness was seen in negative experiences, as noted above where the watch gave too easy workouts. For a digital coach to feel "personalized", users need to actually feel the device identifies their needs correctly. Comparatively, another digital coach study [9] done among athletes there showed a difference. Whereas less active people seem to appreciate the feeling of competence and relatedness provided by the digital coach, more physically active people highlighted the increase in the feeling of autonomy as the main motivational factor related to digital coaching.

Our results also seem to parallel the ideas in the METUX model. The user outcomes - engagement, motivation, and thriving - are intertwined. This was seen, for example, when participants lost motivation as 
a result of feeling less engaged in easy training. One outcome seems to lead to further outcomes.

However, a digital coach as a proxy agent is not necessarily yet as effective or appreciated as a human coach for people with low physical activity levels. Digital coaches still lack a human element that seems to be very important for this target group. Based on the results of this study, digital coaching has potential to teach people how to take control of their own exercising by educating them on their own health. The results also suggest that participants were interested in having a digital coach to increase their own skills and knowledge and to get instruction on how to improve their skills. These findings go together with Bandura's [20] findings related to reasons for using proxy agency. In a previous study [25], having a human proxy agent was seen to lower self-regulatory and task self-efficacy.

Conversely, this study showed that a digital coach may enhance self-regulatory skills and task selfefficacy. Some participants felt they had learned enough from the digital coach and no longer needed a proxy agent at all. However, with the majority of participants still stating they would still choose a human coach over a digital one, there are still qualities a human coach possess that digital coaches have not yet reproduced.

As a practical contribution the study presents implications for future development for digital coaches. Firstly, it seemed that the wrist-based heart rate measurement is not yet accurate enough, which can cause lack in motivation to use the device in the first place. Therefore, it would be important to offer another type of heart rate measurement option along the optical heart rate measurement. It is also important to further personalize the instructional feedback and training guidance to give the users the feeling the digital coach is looking after them. The users should also be offered a possibility to study more about a certain interesting topic which could be done for example via application or computer. Different users are generally interested in different features and therefore the users could be given a possibility to personalize their own digital coach by choosing how it looks and what information it has. These suggestions support the results of Yardley et al. [35] that highlighted the importance of a person-based approach, especially in digital interventions. Also, increasing motivational and supportive messaging could be a way to increase the motivation to continue using the digital coach, especially in the user group of people who are only starting to become more physically active.

Based on the findings the needs and wants related to digital coaching differ for different people. However, the role of educational, supportive and motivational messaging seems to play an important role in this target group irrespective of said needs and wants. Usability issues are also important. Although a digital coach was seen as a relatively inexpensive option, the participants in this target group still preferred a human coach over a digital one. However, based on the results it can be seen that there is an interest in using a combination of digital and personal human coach.

\section{Limitations and future research}

There are a few limitations in the study. Firstly, a less than three-month long study period is relatively short, although the period was long enough for participants to experience how a digital coach can affect their physical activity. Secondly, the average age of university students in Finland can be higher compared to other countries [36] and therefore the participants in this study represented a wide age range. This might make the comparison with studies done in other countries less direct. Thirdly, the results of this study are based on the usage experiences from one particular digital coach device. The results could vary depending on the device/application used in the study. This study is among the first to focus on the user experiences of digital coaching among people with low physical activity. The topic of sport and wellness technology digital coaching continues to be important. Future studies could focus on exploring the topic with different types of digital coaching devices or among different target groups, such as elderly people or teenagers. It could also be interesting to study the possible negative effects of digital coaching on users especially from an exercise psychology point of view.

\section{References}

[1] A. Cocca, J. Liukkonen, D. Mayorga-Vega, and J. VicianaRamírez, "Health-related Physical Activity Levels in Spanish Youth and Young Adults", Perceptual and Motor Skills, 118 (1), 2014, pp. 247-260.

[2] J. D. Irwin, "Prevalence of University Students' Sufficient Physical Activity: A Systematic Review", Perceptual and Motor Skills, 98(3), 2004, pp. 927-943.

[3] World Health Organization (WHO), "Global Strategy on Diet, Physical Activity and Health." 2020, URL: https://www.who.int/dietphysicalactivity/factsheet_inact ivity/en/ (visited on 14/07/2020).

[4] C. Comegys, M. Hannula, and J. Väisänen, "Longitudinal Comparison of Finnish and US Online Shopping Behaviour among University Students: The Five-stage Buying Decision Process", Journal of Targeting, Measurement and Analysis for Marketing, 1(4), 2006, pp. 336-356.

[5] C. Winstein, and R. Schmidt, "Reduced Frequency of Knowledge of Results Enhances Motor Skill Learning", Journal of Experimental Psychology: Learning, Memory, and Cognition, 16, 1990, pp. 677-691.

[6] D. Liebermann, L. Katz, M. Hughes, R. Bartlett, J. McClements and I. Franks, "Advances in the Application 
of Information Technology to Sport Performance", Journal of Sports Sciences, 20(10), 2002, pp. 755-769.

[7] P. Düking, A. Hotho, H-C. Holmberg, F. Fuss and B. Sperlich, "Comparison of Non-invasive Individual Monitoring of the Training and Health of Athletes with Commercially Available Wearable Technologies." Frontiers in Physiology, 7 (71), 2016, pp. 1-11.

[8] S. Halson, J. Peake, and J. Sullivan, "Wearable Technology for Athletes: Information Over-load and Pseudoscience?,", International Journal of Sports Physiology and Performance 11 (6), 2016, pp. 705-706.

[9] T. Kari, S. Koivunen, L. Frank, Makkonen, P. and P. Moilanen. "Critical experiences during the implementation of a self-tracking technology." The proceedings of the 20th PACIS Conference, Chiayi, Taiwan, 2016.

[10] T. Kari, S. Koivunen, L. Frank, M. Makkonen, and P. Moilanen, "The Expected and Perceived Wellbeing Effects of Short-term Self-tracking Technology Use", International Journal of Networking and Virtual Organisations 17(4), 2017, pp. 354-370.

[11] E.A. Locke, and G. P. Latham, "Building a Practically Useful Theory of Goal Setting and Task Motivation: A 35-year odyssey", American Psychologist, 57(9), 2002, pp. 705-717.

[12] M. K. Shilts, M. Horowitz and M.S. Townsend, "Goal Setting as a Strategy for Dietary and Physical Activity Behavior Change: a Review of the Literature", American Journal of Health Promotion, 19(2), 2004, pp. 81-93.

[13] E. Kettunen and T Kari. "Can Sport and Wellness Technology be My Personal Trainer? - Teenagers and Digital Coaching", Proceedings of the 30th Bled eConference, Bled, Slovenia, 2018, pp. 463-476.

[14] B. Schmidt, S. Benchea, R. Eichin and C. Meurisch, "Fitness Tracker or Digital Personal Coach: How to Personalize Training." Proceedings of the ACM International Joint Conference on Pervasive and Ubiquitous Computing and ACM International Symposium on Wearable Computers, Osaka, Japan: ACM, 2015, pp. 1063-1067.

[15] M. Kranz, A. Möller, N. Hammerla, S. Diewald, L. Roalter, T. Ploetz and P. Olivier, "The Mobile Fitness Coach: Towards Individualized Skill Assessment Using Personalized Mobile Devices" Pervasive and Mobile Computing 9, 2013, pp. 203-215.

[16] E. Kettunen, C. Critchley and T. Kari. "Can Digital Coaching Boost Your Performance? - A Qualitative Study among Physically Active People." Proceedings of the 52nd Hawaii International Conference on System Sciences, Maui, USA, 2019.

[17] Bandura, A., Social Foundations of Thought and Action: A Social Cognitive Theory, Englewood Cliffs, NJ: Prentice-Hall, 1986.

[18] A. Bandura, "Health promotion from the Perspective of Social Cognitive Theory", Psychology and Health, 13(4), 1998, pp. 623-649.

[19] R.M. Ryan and E.L. Deci, "Intrinsic and Extrinsic Motivations: Classic Definitions and New Directions", Contemporary Educational Psychology, 25(1), 2000, pp. 54-67.
[20] A. Bandura, "Self-efficacy Mechanism in Human Agency", American Psychologist, 37, 1982, pp. 122-147.

[21] Beauchamp, M. R. and M. A. Eys, Group Dynamics in Exercise and Sport Psychology - Contemporary Themes, New York, NY: Routledge, 2007.

[22] Bandura, A., Self-efficacy: The Exercise of Control. New York, NY: Freeman, 1997.

[23] D. Feltz and C. Lirgg, "Self-efficacy Beliefs of Athletes, Team, and Coaches" In R. Singer, H. Hausenblas and C. Janelle (Eds.) Handbook of Sport Psychology, 2nd ed., New York: John Wiley \& Sons, 2001, pp. 340-361.

[25] C. Shields, and L. Brawley, "Preferring Proxy-agency: Impact on Self-efficacy for Exercise", Journal of Health Psychology, 11(6), 2006, pp. 904-914.

[26] Suunto, 2020, URL:https://www.suunto.com/engb/Products/sports-watches/suunto-3-fitness/suunto-3fitness-all-black/ (visited on 14.7.2020)

[27] UKK Institute, "Physical Activity Pie", 2020, URL: https://www.ukkinstituutti.fi/en/productsservices/physical_activity_pie (visited on 11/07/2020)

[28] Finnish Sports Federation, Kansallinen Liikuntatutkimus 2009-2010: Aikuis- ja senioriliikunta. [National Sports Survey 2009-2010] Report, Finnish Sports Federation, Helsinki, Finland, 2011.

[29] M. Myers, and M. Newman, "The Qualitative Interview In IS Research: Examining The Craft." Information and Organization, 17(1), 2017, pp. 2-26.

[30] V. Braun, and V. Clarke, "Using Thematic Analysis in Psychology", Qualitative Research in Psychology, 3(2), 2006, pp. 77-101.

[31] Guest, G., K.M. MacQueen and E.E. Namey, Applied Thematic Analysis. Los Angeles, CA: SAGE, 2012.

[32] T. Kari, E. Kettunen, P. Moilanen and L. Frank, "Wellness Technology Use in Everyday Life: A Diary Study", Proceedings of the 30th Bled eConference: Bled, Slovenia, University of Maribor, 2017a, pp. 279-294.

[33] J.B. Wang, J.K. Cataldo, G.X. Ayala, L. Natarajan, L.A. Cadmus-Bertram, M.M. White... and J.P. Pierce, "Mobile and Wearable Device Features that Matter in Promoting Physical Activity", Journal of Mobile Technology in Medicine, 5(2), 2016, pp. 2-11.

[34] E. Kettunen, T. Kari, M. Makkonen, W. Critchley and A. Sell, "Digital Coaching among Physically Inactive University Students: A Quantitative Intervention Study on Exercise Self-efficacy." Proceedings of the 32th Bled eConference: Bled, Slovenia, University of Maribor, University of Maribor, 2019, pp. 861-880.

[35] L. Yardley, L. Morrison, K. Bradbury and I. Muller, "The Person-Based Approach to Intervention Development: Application to Digital Health-Related Behavior Change Interventions", Journal of Medical Internet Research, 17(1), 2015, pp. e30.

[36] Finnish National Agency for Education, 2017, URL: https://www.oph.fi/fi/uutiset/2017/oecd-vertailusuomalaiset-suorittavat-opintonsa-loppuun-muttakeskimaaraista

[37] D. Peters, R.A. Calvo, and R.M. Ryan, "Designing for Motivation, Engagement and Wellbeing in Digital Experience.” Frontiers in Psychology, 9(797), pp. 1-15 